\title{
Expression of Matrix Metalloproteinase 3 in Experimental Atherosclerotic Plaques
}

\author{
Yasuhiro Okamoto', Kimio Satomura', Fumitaka Ohsuzu', Haruo Nakamura², Kyoko \\ Takeuchi', and Masahiko Yoshioka'
}

'First Department of Internal Medicine and the First Department of Anatomy, National Defense Medical College, Saitama, Japan.

${ }^{2}$ Mitsukoshi Health and Welfare Foundation, Tokyo, Japan.

\begin{abstract}
In atherosclerotic lesions, matrix metalloproteinases produced by foam cells (macrophages) are thought to increase plaque instability, promote plaque rupture, by degradating extracellular matrix. To investigate the relationship between the expression of these proteinases and the histologic appearance of atheromas, immunohistochemical analysis of matrix metalloproteinase 3 and cell-type markers was performed in atherosclerotic plaques induced in rabbit abdominal aortas by high-cholesterol diets and mechanical injury. In addition to an antibody against matrix metalloproteinase 3, RAM-11 and HHF-35 were used to detect macrophages and smooth muscle cells, respectively. Matrix metalloproteinase 3 was expressed diffusely within the plaques with a fibrofatty histologic pattern. In plaques with foam cell accumulation, matrix metalloproteinase 3 was seen in areas rich in foam cells and the smooth muscle cells near the lumen. In the plaques with fewer macrophages, the proteinase was expressed only in such smooth muscle cells. Matrix metalloproteinase 3 was expressed in the smooth muscle cells in plaques of all histologic types, and macrophages also expressed the metalloproteinase when present in significant numbers. These findings suggest that macrophage accumulation plays an important pathophysiologic role in causing the instability of atherosclerotic lesions by increasing the levels of matrix metalloproteinase $3 . \quad J$ Atheroscler Thromb, 2001 ; 8 : 50-54.
\end{abstract}

Key words : Atherosclerosis, MMP-3, Macrophage, Smooth muscle cell

\section{Introduction}

In atherosclerotic lesions, the excessive production of matrix metalloproteinases (MMP) by smooth muscle cells (SMC) and "foam cells" (macrophages) has been reported (1-8). In particular, MMP produced by macrophages are thought to be related to the instability of atherosclerotic plaques (5-7). The purpose of this immunohistochemical study was to assess the extent to which the production of

Address for correspondence: Yasuhiro Okamoto, First Department of Internal Medicine, National Defense Medical College, 2-3 Namiki Tokorozawa, Saitama 359-8513, Japan. E-mail:PGB02665@nifty.ne.jp

Received June 25, 1999.

Accepted for publication April 4, 2001
MMP by macrophages is likely to contribute to plaque instability.

\section{Materials and Methods}

Induction and observation of atherosclerotic plaques

Two or 3 weeks-old male white rabbits were used. An atheromatous plaque was produced in the rabbit abdominal aorta by the insertion of a polyethylene tube (internal diameter, $0.6 \mathrm{~mm}$; external diameter, $1.2 \mathrm{~mm}$ ) in the aorta for 8 weeks. During the 8 weeks while the tubes were in place, the rabbits were fed commercially available pellets supplemented with $2 \%$ cholesterol to promote plaque formation $(9,10)$. After removal of the tubes, the rabbits were fed with cholesterol-free pellets. Atheromatous plaques were examined 1 month and 6 
months after induction ( $n=5$ for each time point). Aortas from rabbits kept for the same period before observation but not receiving dietary or mechanical atherogenic stimulation were used as controls ( $n=5$ for each time point).

\section{Histologic and immunohistochemical analysis}

Atherogenic lesions were located using an angioscope before portions were excised (10). A portion of each excised aorta was fixed with Bodian solution, embedded in paraffin, serially sectioned into $5 \mu \mathrm{m}$-thick sections, and stained with hematoxylin and eosin. Some of the serial sections were used for immunohistochemical analysis (11). The antibodies used were HHF-35 (Dako, diluted $1: 5,000$ in phosphate-buffered saline (PBS)); RAM-11 (Dako, diluted 1:5,000 in PBS) ; and anti-rabbit MMP-3 protein antibody (Fuji Yakuhin Kogyo, diluted 1:200 in PBS). Biotinylated antiserum to mouse IgG (Dako, diluted 1:200 in PBS) was used as the secondary antibody. Further reaction with labeled streptavidin (Dako, diluted 1: 500 in PBS) and 3,3'-diaminobenzidine (DAB), which showed immunoreactivity as a brown color (11-13). Sections from control aortas were stained similarly using the same histologic and immunohistochemical procedures described above.

\section{Results}

In the control aortas, the media had thick, regularly arranged elastic fibers and SMC with no foam cells. MMP-3 was expressed in the SMC of the media (Fig. 1). One month after injury, marked intimal thickening was noted. In these lesions, a fibrofatty histologic pattern or a marked accumulation of macrophages was typical; both histologic patterns were observed in all five aortas. MMP-3 was diffusely expressed in cells in the lesions with fibrofatty patterns (Fig. 2). In lesions with a marked accumulation of macrophages, MMP-3 was strongly expressed in the macrophages and it was also expressed in the SMC nearest the lumen (Fig. 3). Six months after induction, organization and calcification were seen in the plaque lesions, suggesting progression and remodeling. Fewer macrophages and more SMC was noted in plaques examined 6 months after induction. In such lesions, MMP-3 was expressed mainly in SMC, regardless of the presence of macrophages (Figs. 4, 5).
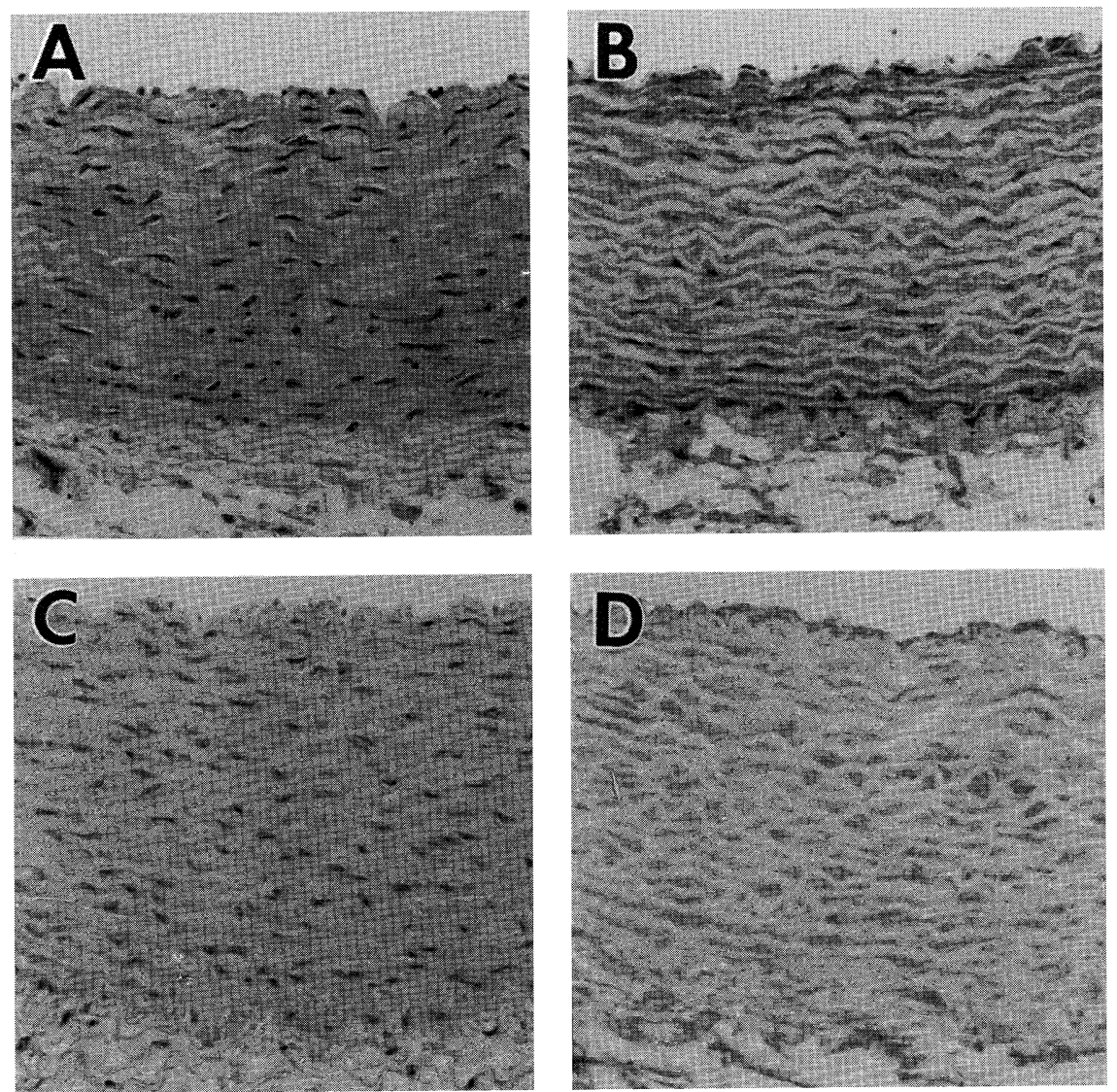

Fig. 1. Immunohistochemical findings in a control aorta. A : hematoxylin and eosin for orientation. B : distribution of smooth muscle cells shown by HHF-35 staining. $\mathrm{C}$ : distribution of macrophages shown by RAM-11. $D$ : matrix metalloproteinase 3 antibody. Magnification is $\times 200$. 

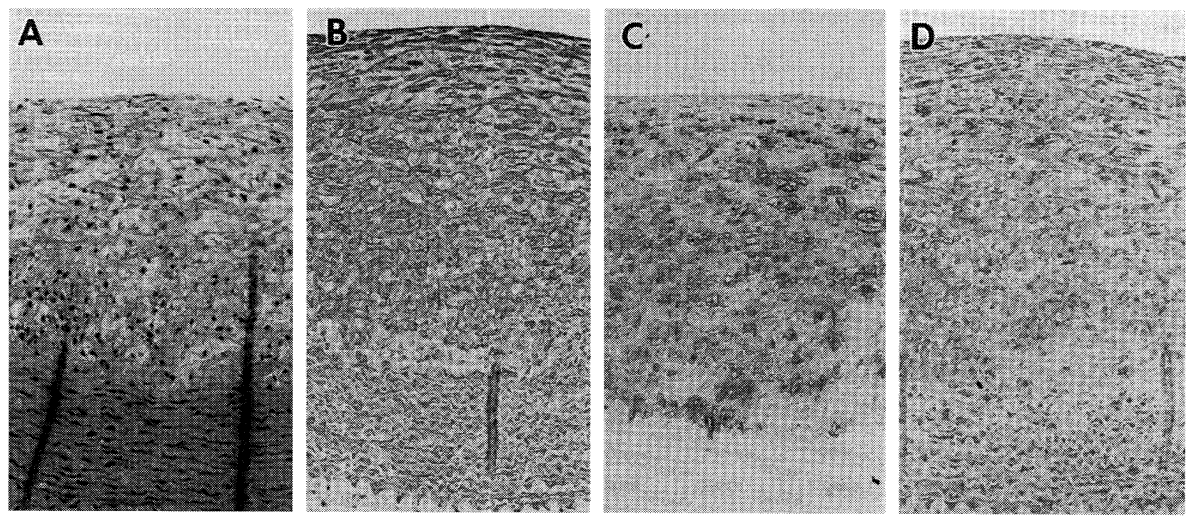

Fig. 2. Immunohistochemical findings in an aorta 1 month after lesion induction without marked macrophage accumulation. A : lesion without marked macrophage accumulation stained by hematoxylin and eosin. B: distribution of smooth muscle cells in the same lesion detected by HHF-35. C : distribution of macrophages in the same lesion detected by RAM-11. D : matrix metalloproteinase 3 revealed in both types of cells without a distinct pattern in same lesion. Magnification is $\times 200$.
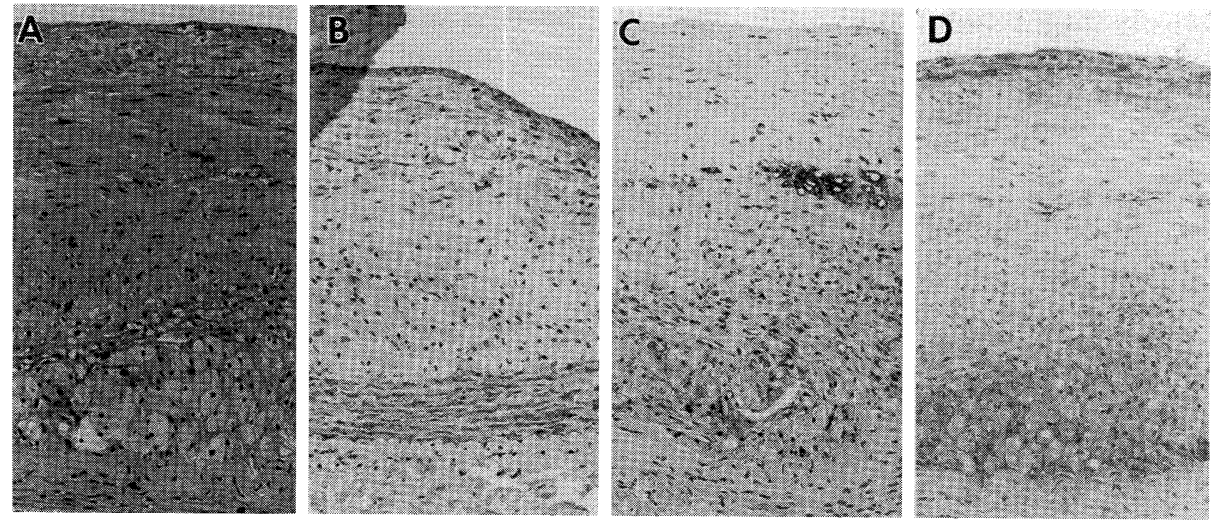

Fig. 3. Immunohistochemical findings in an aorta 1 month after lesion induction with marked macrophage accumulation. A : a lesion with marked macrophage accumulation stained by hematoxylin and eosin. B: distribution of smooth muscle cells in the lesion A detected by HHF-35. C : distribution of macrophages in the lesion shown in A (RAM-11). D: matrix metalloproteinase 3 shown in accumulating macrophages and SMC nearest the lumen in the lesion A. Magnification is $\times 200$.
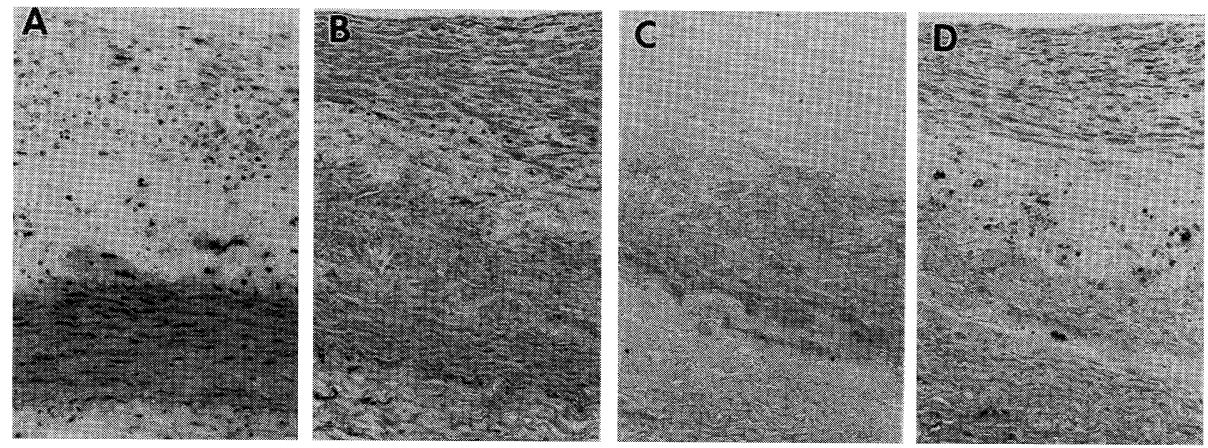

Fig. 4. Immunohistochemical findings in an aorta 6 months after lesion induction without macrophage accumulation. A : a lesion 6 months after induction; macrophage accumulation is not seen (hematoxylin and eosin). B : distribution of smooth muscle cells in the same lesion detected by HHF-35. C : distribution of macrophages in the same lesion detected by RAM-11. D : matrix metalloproteinase 3 is seen in smooth muscle cells in the lesion A. Magnification is $\times 200$. 

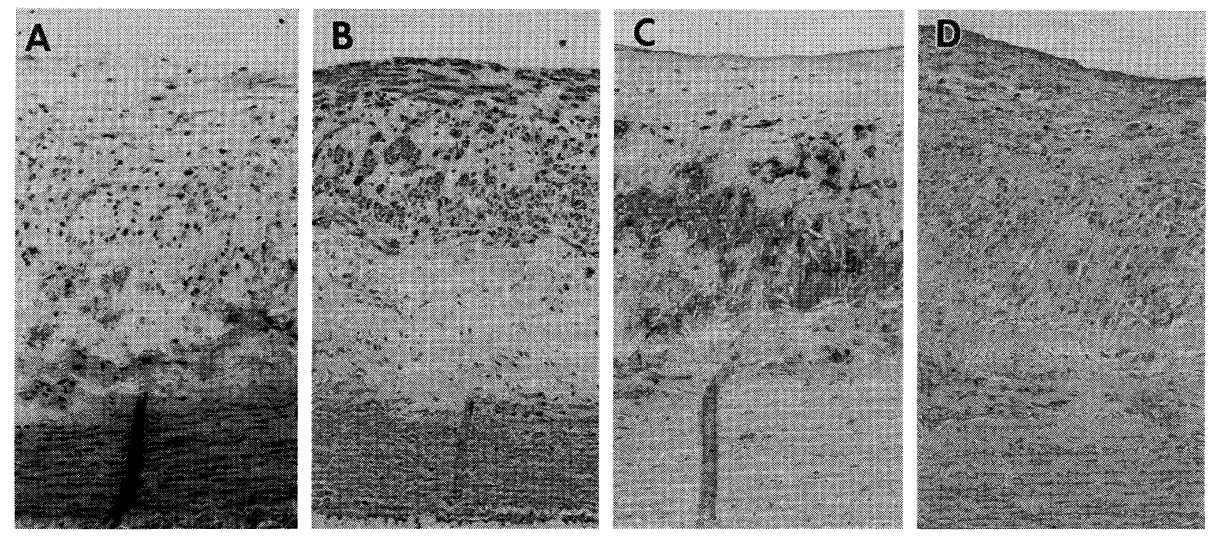

Fig. 5. Immunohistochemical findings in an aorta 6 months after induction with macrophage accumulation. A : a lesion with more markedly petrified macrophages (hematoxylin and eosin). B : distribution of smooth muscle cells in the lesion A (HHF-35). C : distribution of macrophages in the lesion in A (RAM-11). D: matrix metalloproteinase 3 is largely restricted to smooth muscle cells in the lesion shown in A. Magnification is $\times 200$.

\section{Discussion}

In atherosclerotic lesions, enhanced MMP production by $\mathrm{SMC}$ is reported to occur in response to certain cytokines or mechanical injury (1-3). MMP produced by macrophages in atherosclerotic lesions has been related to plaque instability (4-7). In this study, the production of MMP-3 was assessed in both macrophages and SMC in various histologic types of atherosclerotic lesions.

MMP-3 was expressed by SMC in all histologic patterns of atherosclerotic plaque, as well as in the control aortas without atherosclerotic lesions. Macrophages strongly expressed MMP-3 when present in clusters.

Since various types of MMP are produced by SMC in the course of development (14), SMC production of MMP is thought to maintain normal vascular structure despite various insults. In contrast, instability of the atherosclerotic lesion might be affected mainly by MMP produced by macrophages in clusteration (15).

Although our study is limited to preliminary findings, considering that MMP-3 is the most potent activator of other MMP (16), we consider that our findings should be helpful in elucidating the pathophysiologic roles of MMP from SMC and macrophages in atherosclerosis.

The therapeutic implications of the handling plaque stability might be enhanced by the inhibition of macrophages clusteration or MMP production by macrophages should be investigated in the future $(15,17)$.

\section{References}

(1) Yanagi H, Sasaguri $Y$, Suganuma K, Morimatsu M, and Nagase $H$ : Production of tissue collagenase (matrix metalloproteinase 1) by human aortic smooth muscle cells in response to platelet-derived growth factor. Atherosclerosis, 91 : 207-216, 1992

(2) Boteny MD, Kaiser LR, Cooper JD, Mecham RP, Parghi D,
Roby J, and Parks WC: Extracellular matrix protein gene expression in atherosclerotic hypertensive pulmonary arteries. Am J Pathol, 140: 357-364, 1992

(3) Nikkari ST, Jarvelainen HT, Wight TN, Ferguson M, and Clowes AW : Smooth muscle cell expression of extracellular matrix genes after arterial injury. Am J Pathol, 144 : 1348-1356, 1994

(4) Galis ZS, Sukhova GK, Lark MW, and Libby P: Increased expression of matrix metalloproteinases and matrix degrading activity in vulnerable regions of human atherosclerotic plaques. J Clin Invest, 94 : 2493-2503, 1994

(5) Galis ZS, Sukhova GK, and Libby P: Microscopic localization of active proteases by in situ zymography : Detection of matrix metalloproteinase activity in vascular tissue. FASEB J, 9: 974-980, 1995

(6) Galis ZS, Sukhova GK, Krazhoffer R, Clark S, and Libby $P$ : Macrophage foam cells from experimental atheroma constitutively produce matrix-degrading proteinase. Proc Natl Acad Sci USA, 92 : 402-406, 1995

(7) Halpert I, Sires UI, Roby JD, Potter-Perigo S, Wight TN, Shapiro SD, Welgus HG, Wickline SA, and Parks WC: Matrilysin is expressed by lipid-laden macrophages at sites of potential rupture in atherosclerotic lesions and localizes to areas of versican deposition, a proteoglycan substrate for the enzyme. Proc Natl Acad Sci USA, 93: 9748-9753, 1996

(8) Knox JB, Sukhova GK, Whittemore AD, and Libby P: Evidence for altered balance between matrix metalloproteinases and their inhibitors in human aortic diseases. Circulation, 95: 205-212, 1997

(9) Bocan TM, Mueller SB, Uhlendorf PD, Ferguson E, and Newton RS: Dietary and mechanically induced rabbit iliac-femoral atherosclerotic lesions. Exp Mol Pathol, 54 : 201-277, 1991

(10) Okamoto Y, Mizuno K, Arakawa K, Nakamura H, Takeuchi $\mathrm{K}$, and Yoshioka $\mathrm{M}$ : In vivo angioscopic detection of the damaged endothelium on the atheromatous plaque. Am J Cardiac Imag, 9: 57-61, 1995

(11) Okamoto $Y$ : Detection of damaged endothelium on the experimental atheromatous plaque and histo-pathological analysis of the plaque (II). J. Natl. Def. Med. Coll, 
(Journal of National Defense Medical College) $20: 22$ 28, 1995 (Japanese with English abstract)

(12) Stadius ML, Rowan R, Fleischhauer F, Kernoff R, Billingham $M$, and Gown $A M$ : Time course and cellular characteristics of the iliac artery response to acute balloon injury. Arterioscler Thromb, 12:1267-1273, 1992

(13) Katsuda S, Boyd HC, Flinger C, Ross R, and Gown AM: Human atherosclerosis (III). Immunohistochemical analysis of the cell composition of lesions of young adults. Am J Pathol, 140: 907-914, 1992

(14) Sasaguri $Y$, Murahashi N, Sugama K, Kato S, Hiraoka K, Satoh $\mathrm{T}$, Isomoto $\mathrm{H}$, and Morimatsu $\mathrm{M}$ : Developmentrelated changes in matrix metalloproteinases expression in human aortic smooth musle cells. Lab Invest, 71 : 261-269, 1994

(15) Aikawa M, Rabkin E, Okada Y, Voglic SJ, Clinton SK, Brickerhoff CE, Sukhova G, and Libby P: Lipid lowering by diet reduces matrix metalloproteinase activity and increases collagen content of rabbit atheroma. Circulation, 97 : 2433-2444, 1998

(16) George SJ: Tissue inhibitors of metalloproteinases and metalloproteinases in atherosclerosis. Curr Opin Lipidol, 9 : 413-423, 1998

(17) Galis ZS, Asanuma K, Godin D, and Meng X: N-acetylcystein decreases the matrix-degrading capacity of macrophage-derived foam cells. Circulation, 97 : 2445-2453, 1998 